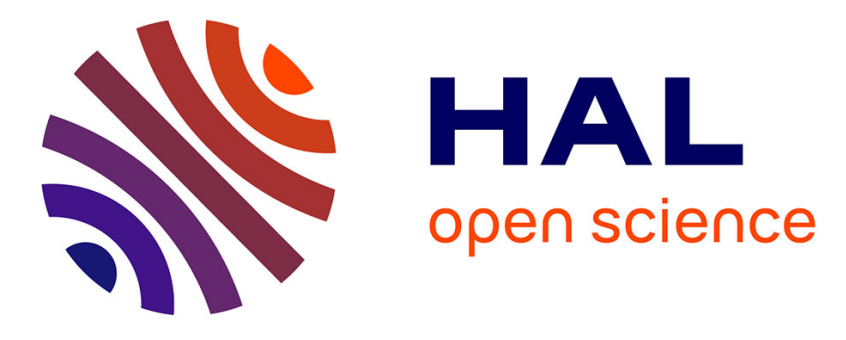

\title{
Réalisation d'un caisson hermétique pour essais dynamiques
}

J. Petit, A. Nadeau, M. C. Lafarie, Narayanaswami Ranganathan

\section{To cite this version:}

J. Petit, A. Nadeau, M. C. Lafarie, Narayanaswami Ranganathan. Réalisation d'un caisson hermétique pour essais dynamiques. Revue de Physique Appliquée, 1980, 15 (4), pp.919-923. 10.1051/rphysap:01980001504091900 . jpa-00244805

\section{HAL Id: jpa-00244805 https://hal.science/jpa-00244805}

Submitted on 1 Jan 1980

HAL is a multi-disciplinary open access archive for the deposit and dissemination of scientific research documents, whether they are published or not. The documents may come from teaching and research institutions in France or abroad, or from public or private research centers.
L'archive ouverte pluridisciplinaire HAL, est destinée au dépôt et à la diffusion de documents scientifiques de niveau recherche, publiés ou non, émanant des établissements d'enseignement et de recherche français ou étrangers, des laboratoires publics ou privés. 


\title{
Réalisation d'un caisson hermétique pour essais dynamiques
}

\author{
J. Petit, A. Nadeau, M. C. Lafarie et N. Ranganathan \\ E.R.A. 123, E.N.S.M.A., 86034 Poitiers, Cedex, France
}

(Reçu le 24 octobre 1979, révisé le 26 décembre 1979, accepté le 2 janvier 1980)

1

Résumé. - Un nouveau dispositif d'essais dynamiques sous vide ou sous atmosphère contrôlée a été réalisé. Cet ensemble est monté sur le bâti d'une machine hydropulse de 2500 daN et de $100 \mathrm{~mm}$ de course. L'étanchéité est assurée à l'aide d'un soufflet métallique. Les essais de fatigue réalisés montrent d'une part la fiabilité du dispositif adopté, d'autre part la facilité et la souplesse d'emploi de ce caisson pour étudier les effets d'environnement en permettant d'obtenir rapidement un vide meilleur que $5 \times 10^{-3} \mathrm{~Pa}$ ou d'établir un environnement gazeux de $100 \mathrm{~Pa}$ à $2 \times 10^{5} \mathrm{~Pa}$.

\begin{abstract}
A new dynamic testing system under vacuum or controlled environment had been realized. It has been mounted on the frame of an electro-hydraulic machine (capacity :2 500 daN, stroke : $100 \mathrm{~mm}$ ). The tightness is obtained with metallic bellows. The tests which had been carried out, confirm the fiability of this system and the ease with it can be utilized to study the effect of environment at a vacuum of less than $5 \times 10^{-3} \mathrm{~Pa}$ or any other gas at a controlled pressure of $10^{2}-2 \times 10^{5} \mathrm{~Pa}$.
\end{abstract}

1. Introduction. - L'étude des conditions d'amorçage et de propagation des fissures de fatigue dans les pièces mécaniques et les structures a fait des progrès importants au cours des vingt dernières années. Les deux raisons essentielles sont d'une part l'apparition sur le marché de machines d'essai électrohydrauliques asservies qui permettent de maintenir les paramètres mécaniques avec une bonne précision et d'autre part l'utilisation des concepts et des méthodes de la mécanique de la rupture donnant les moyens de quantifier ces mêmes paramètres.

L'un des résultats récents a été de montrer que la propagation d'une fissure de fatigue est fortement influencée par la nature de l'atmosphère ambiante [1-4]. Non seulement un environnement corrosif peut réduire de façon importante la durée de vie. mais l'air du laboratoire donne déjà des vitesses de propagation nettement supérieures à celles qu'on obtient sous vide ou sous atmosphère parfaitement neutre [5-6]. Bien que l'existence de ce phénomène soit maintenant bien établie, qualitativement de nombreuses études sont encore nécessaires pour en dégager les explications physiques. De plus, sur le plan quantitatif, les résultats sont encore assez peu concluants; en effet les études ont été faites dans des conditions rarement comparables, en particulier en ce qui concerne la pression partielle ou résiduelle des différents éléments gazeux.

Nous nous sommes donc proposés de construire un dispositif d'essai permettant de solliciter en fatigue des éprouvettes de dimensions convenables, sous un vide aussi poussé que possible, tout en conservant toutes les possibilités d'une machine d'essai électrohydraulique asservie.

2. Description de l'appareillage. - Cet ensemble a été conçu pour répondre à un usage spécifique. Nous avons en particulier cherché à satisfaire les caractéristiques suivantes :

1) adaptation du caisson sur un bâti INSTRON 1271 équipé d'un vérin ayant une capacité dynamique de $\pm 2500 \mathrm{daN}$ et une course de $\pm 50 \mathrm{~mm}$;

2) étanchéité assurée au niveau du vérin par un dispositif conduisant à un effort dû au vide qui soit facile à étalonner et bien reproductible ;

3) obtention assez rapide d'un vide secondaire $<$ à $10^{-3} \mathrm{~Pa}$;

4) emploi aisé d'éprouvettes compactes de type CT selon la norme ASTM ;

5) détermination optique de la longueur de fissure la plus précise possible et d'emploi facile ;

6) prévision des entrées (et sorties) étanches nécessaires aux mesures susceptibles d'être effectuées en cours d'essai :

- mesure de longueur de fissure par variation de potentiel ; 
- mesure de vitesse de propagation par jauge à fils ;

- mesure du bâillement par capteur de déplacement ;

- mesúre de COD par jauges de déformation;

- contrôle de température ;

- mesure de vide (primaire et secondaire);

- vanne permettant d'établir un environnement gazeux contrôlé.

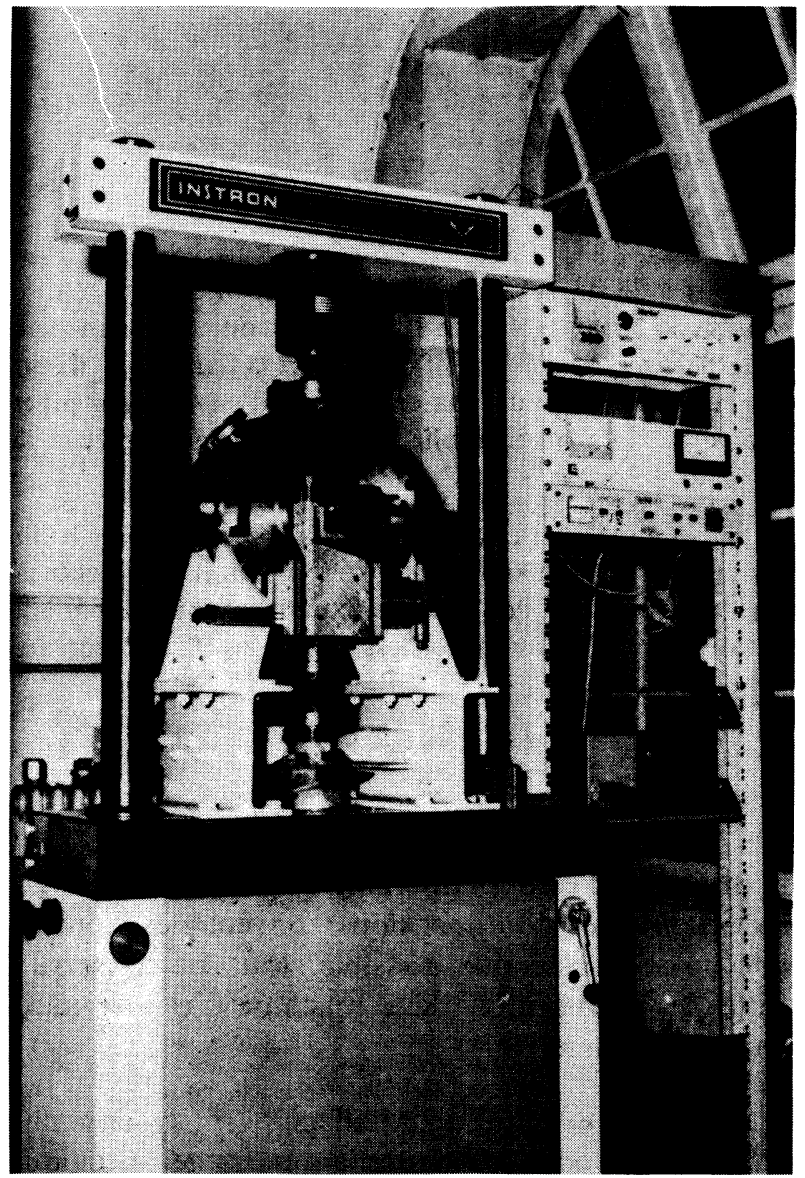

Fig. 1. - Vue d'ensemble.

[Over all view.]
Pour parvenir à ce résultat, nous avons adopté le principe d'un caisson de $300 \mathrm{~mm}$ de diamètre (Fig. 1), constitué d'une virole supportant les passages étanches les plus importants, sur laquelle est soudé un fond bombé avec des passages étanches de plus petites dimensions, et qui laisse en son centre un passage de $150 \mathrm{~mm}$ de diamètre pour le pompage dont le système a été volontairement surdimensionné dans le but d'améliorer les conditions de mise sous vide.

Un couvercle concave, aisément démontable, permet d'observer la surface de l'échantillon à moins de $60 \mathrm{~mm}$ à travers un hublot (Fig. 2).

Les arbres de transmission des efforts qui supportent les mors sont excentrés par rapport au caisson (Fig. 1) afin de déporter les échantillons asymétriques de type CT, ce qui permet une observation optique aisée de la progression des fissures tout en limitant le volume de l'enceinte; il reste cependant une marge suffisante pour observer un échantillon centré sur les mors.

L'étanchéité au niveau de l'arbre relié au vérin est assurée par un soufflet métallique de 22 spires (Fig. 3). Au niveau de l'arbre de mesure elle est assurée par une simple flasque élastique en caoutchouc adapté au vide.

Enfin la lunette assurant le suivi de la fissure (grandissement 25), est installée sur un support solidaire du caisson que l'on peut dégager au moment de l'ouverture par un système à charnière. Un chariot micrométrique à deux axes permet de positionner la lunette au $1 / 100$ de $\mathrm{mm}$ près sur $63 \mathrm{~mm}$ de course horizontale et $16 \mathrm{~mm}$ de course verticale (Fig. 2).

3. Performances obtenues. - 3.1 VIDE. - Le groupe de pompage est équipé d'un piège à azote liquide, et l'ensemble permet d'atteindre dans les meilleures conditions un vide limite inférieur à $2 \times 10^{-5} \mathrm{~Pa}$. Sans utiliser le piège on obtient $6 \times 10^{-5} \mathrm{~Pa}$ en $48 \mathrm{~h}$ environ avec tous les fils de mesure.

Toutefois l'objectif premier est de.pouvoir des-
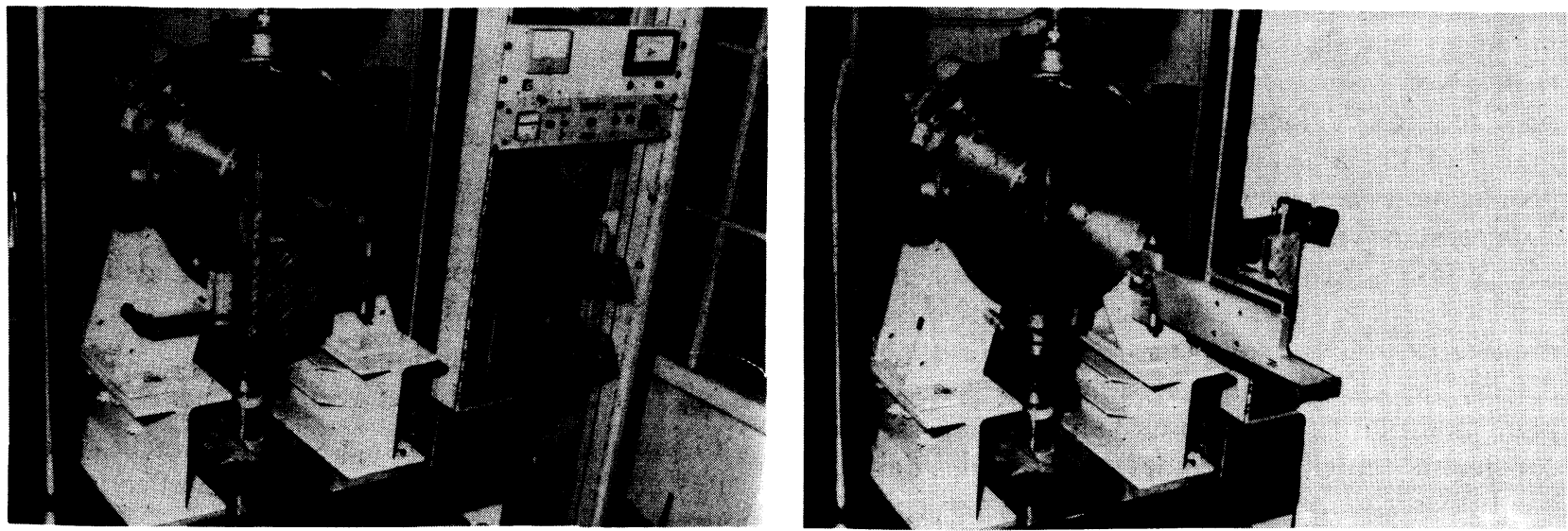

Fig. 2. - Vue du chariot micrométrique : - position fermée ; - position ouverte

[View» of the micrometric carriage : closed position and opened position.] 


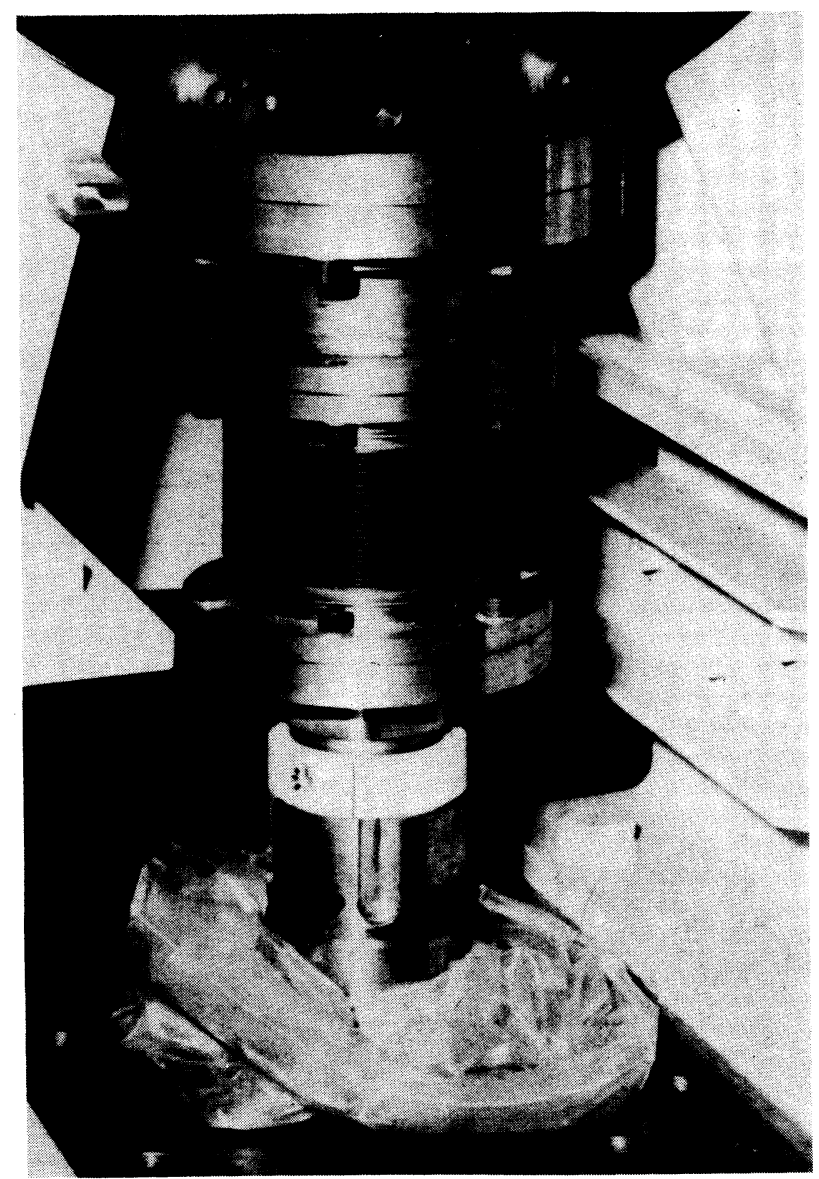

Fig. 3. - Vue du soufflet.

[View of bellows.]

cendre en dessous de $5 \times 10^{-3} \mathrm{~Pa}$ sans précaution particulière. Les pompes étant préchauffées cette valeur est obtenue sans piège en 12 min de pompage, et en moins de $45 \mathrm{~min}$ le vide est inférieur à $10^{-3} \mathrm{~Pa}$.

3.2 Mesure Des efFORTS. - La compacité de l'ensemble lui confère une grande rigidité et les déformations subies à la mise sous vide sont de ce fait très faibles et restent négligeables.

Par ailleurs, la nature élastique des liaisons entre le caisson et les arbres des mors élimine pratiquement les efforts parasites. Ainsi la reproductibilité de l'effet de mise sous vide s'avère-t-elle très bonne et se traduit uniquement par un effort constant sur l'arbre de mesure (12 daN).

La technique adoptée a toutefois un aspect limitatif ; en effet, le soufflet n'autorise pas d'essais prolongés à certaines fréquences (en particulier entre $40 \mathrm{~Hz}$ et $50 \mathrm{~Hz}) \mathrm{du}$ fait de l'apparition de phénomène de résonance qui conduirait rapidement à sa détérioration. Un soufflet plus court est toutefois prévu pour des essais devant impérativement être effectués à ces fréquences (course $10 \mathrm{~mm}$ ).

Des mors adaptables permettent d'utiliser des éprouvettes ayant jusqu'à $40 \mathrm{~mm}$ d'épaisseur et $150 \mathrm{~mm}$ de large. De plus, un montage a été conçu pour solliciter des éprouvettes KT en flexion quatre points.

3.3 Mesure optiQue. - Le dispositif de suivi optique s'est avéré pratique et précis; un réticule réglable dans l'oculaire permet un réglage de zéro et l'on obtient alors directement la valeur du paramètre a sur les éprouvettes CT (distance entre l'axe de sollicitation et le fond de fissure).

Par ailleurs, le hublot de $100 \mathrm{~mm}$ de diamètre étant proche de la surface des éprouvettes, les conditions d'éclairage peuvent être bien maîtrisées (stroboscope, fibres optiques, lumière diffuse), ce qui est souvent très important pour situer avec certitude l'extrémité de la fissure.

4. Exemple d'essais. - 4.1 INFLUENCE DU RAPPORT DE CHARGE ET DE L'ÉPAISSEUR SUR LA PROPAGATION D'UNE FISSURE A L'AIR OU SOUS VIDE, SUR UN ALLIAGE LÉGER TYPE AZ5GU (7075 T651). - Les figures $4 a$ et $4 b$ comparent les courbes $\log \mathrm{d} a / \mathrm{d} N$ en fonction de $\log K_{\mathrm{M}}(\mathrm{d} a / \mathrm{d} N$ vitesse de fissuration en $\mathrm{mm} /$ cycle, $K_{\mathrm{M}}$ facteur d'intensité de contrainte à la charge maximale $P_{\mathrm{M}}$ ) établies à $20 \mathrm{~Hz}$ à l'air et sous vide, pour deux valeurs du rapport de charge $R=P_{\mathrm{m}} / P_{\mathrm{M}} \quad\left(P_{\mathrm{M}}\right.$ charge maximale et $P_{\mathrm{m}}$ charge minimale de la sollicitation ondulée) et deux épaisseurs d'échantillons CT 75 , à savoir $4 \mathrm{~mm}$ et $40 \mathrm{~mm}$. On remarque que pour un $K_{\mathrm{M}}$ donné $\mathrm{d} a / \mathrm{d} N$ diminue lorsque $R$ augmente.

L'effet de l'épaisseur n'est vraiment sensible que pour $R=0,5$ et $t=4 \mathrm{~mm}$.

Ceci est généralement attribué d'une part au fait que l'endommagement devient plus faible quand $R$ croît et que l'effet de bord conduisant à une propagation plus rapide est plus sensible sur les éprouvettes minces $[6,7]$.

Par ailleurs la vitesse de propagation à l'air est toujours plus élevée que sous vide, l'origine de cette différence demeurant un de nos principaux sujets de recherche.

4.2 EFfeT D'UNe SURChARGe. - Lorsque l'on applique une surcharge à une éprouvette au cours d'une fissuration à charge constante, on observe généralement un retard de propagation comme le montrent les figures $5 a$ et $5 b$ correspondant respectivement à des essais à l'air et sous vide sur l'alliage $2024 \mathrm{~T} 351$, (essais $V_{3}$ avec une seule surcharge, essais $A_{2}$ et $V_{2}$ avec quatre surcharges successives).

Le taux de surcharge $\tau=\frac{P_{\mathrm{s}}-P_{\mathrm{m}}}{P_{\mathrm{M}}-P_{\mathrm{m}}}$ est égal à 2 ( $P_{\mathrm{s}}$ charge atteinte au cours du cycle de surcharge, $P_{\mathrm{M}}$ charge maximale et $\boldsymbol{P}_{\mathrm{m}}$ charge minimale au cours de la sollicitation à charge constante). Ici $P_{\mathrm{M}}=660 \mathrm{daN}$ et le rapport de charge $R=P_{\mathrm{m}} / P_{\mathrm{M}}=0,1$.

On voit que l'effet des surcharges se manifeste de façon analogue pour les deux environnements, les retards sous vide étant toutefois beaucoup plus importants ; ainsi :

- quatre surcharges à l'air (essai $A_{2}$ ) multiplient 

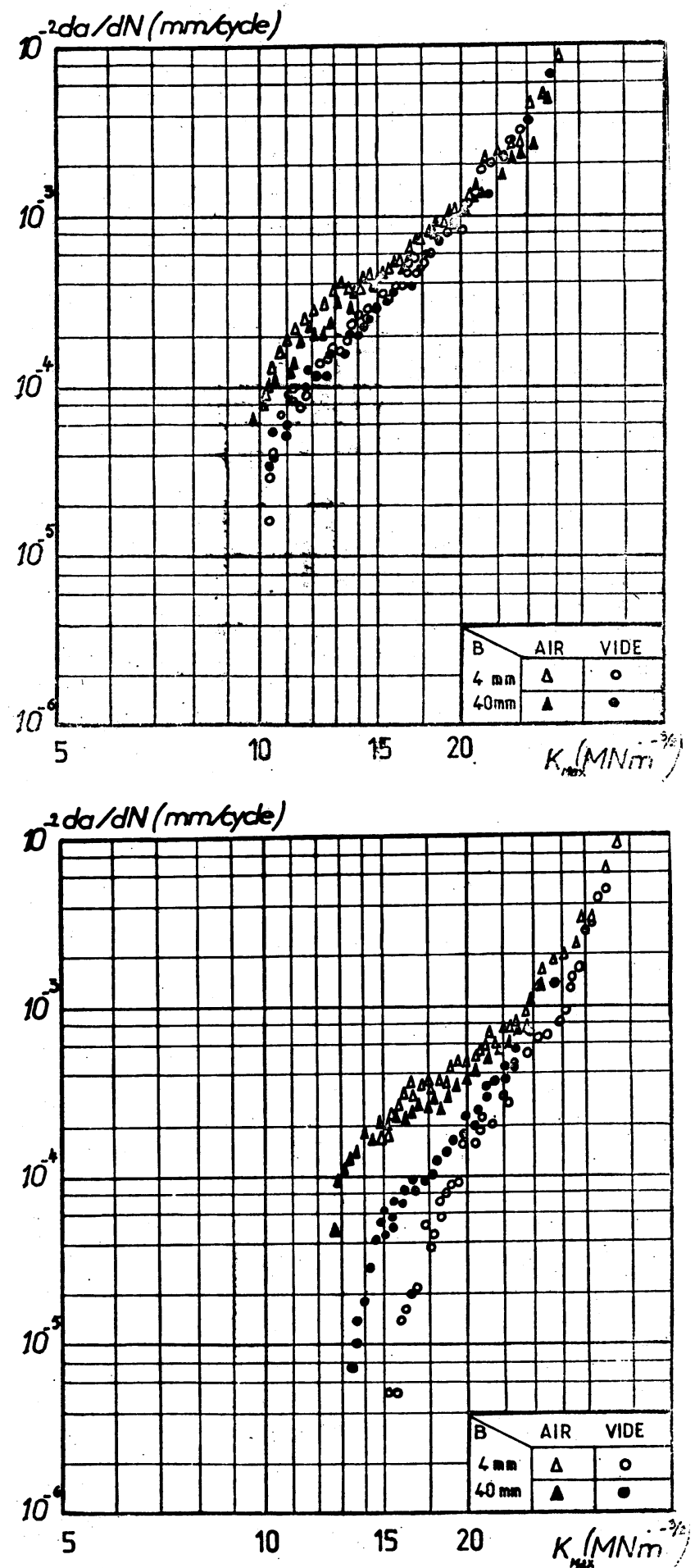

Fig. 4. - Influence de l'épaisseur $B$ et du rapport $R$ sur la vitesse de fissuration de l'alliage 7075 T6.

[Influence of the thickness $B$ and of the load ratio $R$ on the crack propagation rate on the aluminium alloy $7075 \mathrm{~T} 6$.]

Fig. 6. - Comparaison de la vitesse de propagation à l'air (•) et sous azote $(+)$ sur l'alliage 2024 T351 (éprouvettes CT, charge constante, $R=0,1$ ).

[Comparison of crack propagation rates in air $(\bullet)$ and in nitrogen $(+)$ of the aluminium alloy $2024 \mathrm{~T} 351$ (CT specimens, constant $\Delta P$ tests with $R=0.1$.]
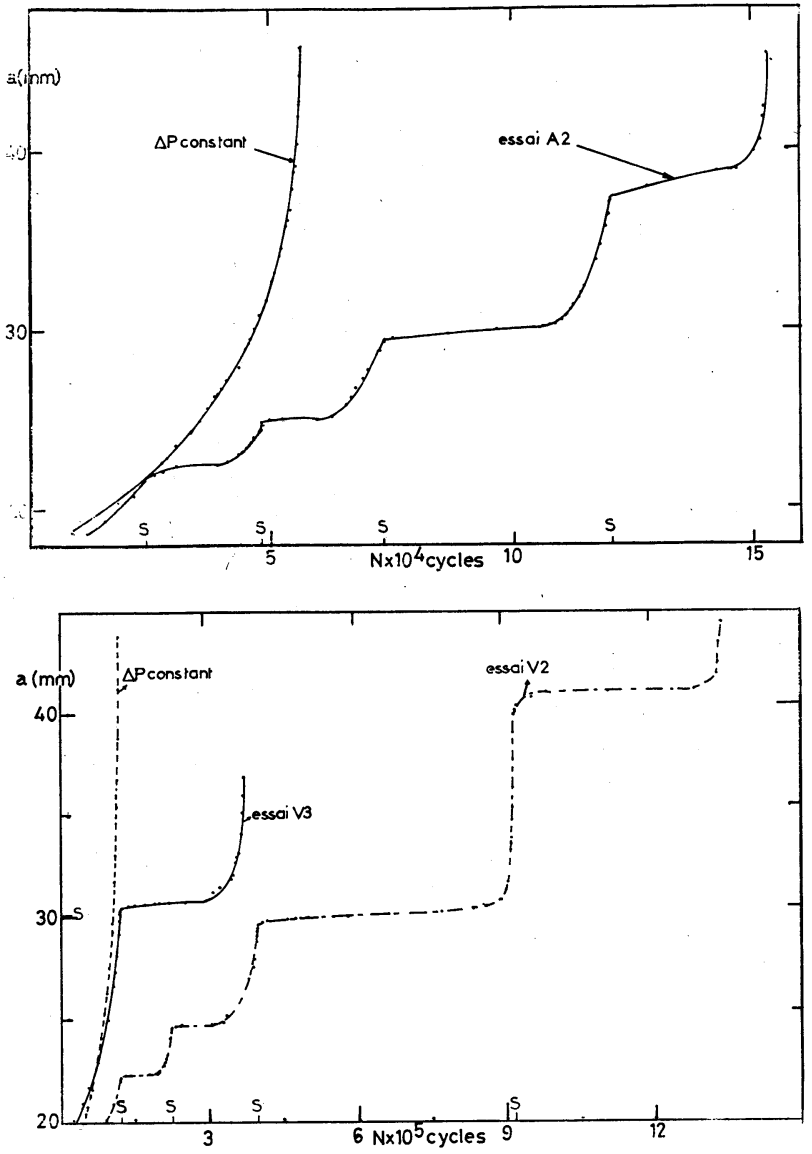

Fig. 5. - Effet de surcharge. Comparaison des courbes de propagation sans surcharge $(\Delta P$ constant) et avec surcharge, $R=0,1: a)$ essais sous air; $\mathrm{A}_{2}$ : quatre surcharges successives ; $b)$ essais sous vide : $V_{3}:$ une seule surcharge $; V_{2}:$ quatre surcharges successives.

[Effect of overload : comparison of crack propagation curves for constant $\Delta P$ tests and overload tests $(R=0.1): a)$ Test in air $\mathrm{A}_{2}$ with four successives overloads. $b$ ) Tests in vacuum : $\mathrm{V}_{2}$ with four successive overloads, $V_{3}$ single overload test.]

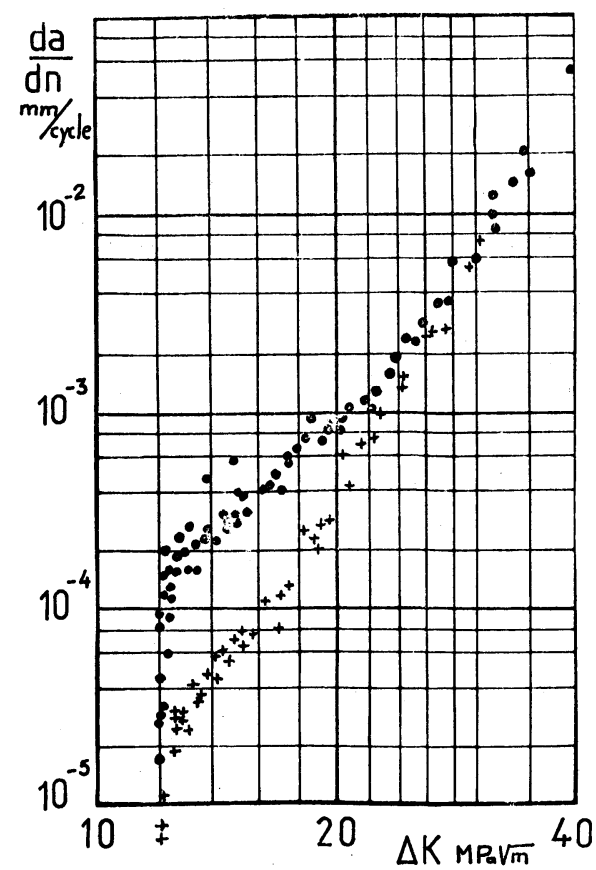


la durée de vie par rapport à un essai sans surcharge par 3 , et quatre surcharges sous vide (essai $V_{2}$ ) la décuplent;

- d'autre part la durée de vie sous vide après ces quatre surcharges est 8 fois plus grande qu'après 4 surcharges à l'air. L'étúde de ces phénomènes est actuellement développée dans le laboratoire [8].

4.3 ESSAI sous ENVIRONNEMENT Gazeux. - Pour illustrer les possibilités de mesure sous environnement contrôlé, une atmosphère d'azote a été établie à l'intérieur de l'enceinte à une pression de 1,2 atm. Les essais comparatifs de fissuration à l'air et sous azote sur l'alliage léger 2024 T351 ainsi effectués montrent (Fig. 6) que la propagation est bien plus lente sous environnement neutre qu'à l'air ambiant dans le domaine de vitesse considéré ; cette différence est généralement attribuée à la présence de vapeur d'eau dans l'air [4].

Toutefois, à l'approche de la rupture finale, les courbes de propagation tendent à se confondre.
Des essais comparatifs entre l'air, l'azote et le vide sont en cours de développement en particulier dans le domaine des basses vitesses.

5. Conclusions. - Les essais réalisés à ce jour sur cet équipement permettent d'affirmer que l'objectif initialement fixé a été atteint. Le caisson permet en effet d'opérer aisément sous un vide inférieur à $10^{-3} \mathrm{~Pa}$ ou sous atmosphère contrôlée sans incidence particulière sur le fonctionnement de la machine hydropulse. La seule limitation concerne certains intervalles de fréquences à éviter (en particulier vers $45 \mathrm{~Hz}$ ) le soufflet entrant en résonance. Par ailleurs le temps effectif de fonctionnement du soufflet atteint à ce jour (supérieur à $2000 \mathrm{~h}$ ), montre la fiabilité du système adopté.

Cet équipement permet donc d'élargir le champ des études déjà engagées au laboratoire sur les équipements existants préalablement [9].

\section{Bibliographie}

[1] Gough, H. J., Sopwith, D. G., J. Inst. Metals 492 (1932) 92-112.

[2] Snonden, K. U., Acta Met. 12 (1964) 295.

[3] Hordon, M. J., Acta Met. 14 (1966) 1173.

[4] Bouchet, B., Thèse Doct. ès Sc. (1971) Poitiers.

[5] Boisson, P., Petit, J., Gasc, C., Mem. Sci. Rev. Met. (1977) 427.
[6] Petit, J., Bouchet, B., Gasc, C. et De Fouquet, J., Proceeding of ICF4. Fracture 2 (1977) 867-871.

[7] Lafarie Frenot, M. C., Thèse 3e cycle (1978) Poitiers.

[8] Ranganathan, N., Petit, J. and Bouchet, B., Eng. Fract. Mech. 11 (1979) 775-789.

[9] Bouchet, B., Petit, J., Chaumet, H., Nadeau, A. et Boisson, P., Mec. Mat. Elec. 327 (1977) 29. 